\title{
MOVIE RECOMMENDATION USING KNOWLEDGE GRAPH
}

\author{
Navin Tatyaba Gopal \\ P.G. Student, Information Security, COEP India
}

Anish Raj Khobragade

Prof. Information Security, COEP India

Article DOI: https://doi.org/10.36713/epra6692

DOI No: 10.36713/epra6692

\begin{abstract}
The Knowledge graphs (KGs) catches structured data and relationships among a bunch of entities and items. Generally, constitute an attractive origin of information that can advance the recommender systems. But, present methodologies of this area depend on manual element thus don't permit for start to end training. This article proposes, Knowledge Graph along with Label Smoothness (KG-LS) to offer better suggestions for the recommender Systems. Our methodology processes user-specific entities by prior application of a function capability that recognizes key KGrelationships for a specific user. In this manner, we change the KG in a specific-user weighted graph followed by application of a graph neural network to process customized entity embedding. To give better preliminary predisposition, label smoothness comes into picture, which places items in the KG which probably going to have identical user significant names/scores. Use of, label smoothness gives regularization above the edge weights thus; we demonstrate that it is comparable to a label propagation plan on the graph. Additionally building-up a productive usage that symbolizes solid adaptability concerning the size of knowledge graph. Experimentation on 4 datasets shows that our strategy beats best in class baselines. This process likewise accomplishes solid execution in cold start situations where user-entity communications remain meager.
\end{abstract}

\section{INTRODUCTION}

Recommender frameworks are generally utilized in Internet applications to meet user customized interests and reduce data surplus. Conventional recommender frameworks which are based on collaborative filtering typically experience cold-start issue and experience trouble suggesting fresh items that haven't yet been intensely searched by the users. The problem can be overcome by presenting extra sources of data like user/entity profiles or else social networking communities. KGs collect organized data and relations among a bunch of elements. These are heterogeneous diagrams in which nodes compare to elements (e.g., elements or items, and its properties and qualities) while edges compare to relations. Knowledge Graphs supply connectivity data among elements by means of various kinds of relations and subsequently catch semantic likeliness among elements. The major 


\section{SJIF Impact Factor 2021: 8.013| ISI I.F.Value:1.241| Journal DOI: 10.36713/epra2016 ISSN: 2455-7838(Online) EPRA International Journal of Research and Development (IJRD)}

task in using knowledge graphs in recommender frameworks is to figure out in what way to catch userspecific element-element likeliness caught by knowledge graph. Prevailing knowledge graph mindful recommender frameworks can be arranged into pathbased techniques, implanting based strategies and hybrid strategies. But, these methodologies depend on manual element, which can't perform start-to-finish point training, and have very low versatility. Graph Neural Network (GNN), which collect data from local nodal network from nearby area utilizing neural networks, address an optimistic headway in graph-built learning. In recent times, few works created GNNs engineering for recommender frameworks yet these methodologies are generally intended for homogeneous bipartite use-element communication graphs or-else user-element similarity graphs. It's an open inquiry in what way we can stretch GNNs engineering to heterogeneous information graphs. The article, described the development of KG-LS that stretches GNNs engineering to KGs to concurrently seize semantic connections among the entities along with customized user inclinations and interests. To represent the social heterogeneity in knowledge graphs, like, we utilize a trainable and customized connection scoring capacity that changes the knowledge graph in a clientexplicit weighted chart that portrays the semantic data of knowledge graph also the client's customized requirements. For instance, in a film recommendation setting a connection scoring capacity might discover that a particular client truly thinks often about "director" connection among movies and persons, while another person may think often more of a "lead actor" connection. By use of this customized weighted graph, at that point we can apply a GNN that for each element node registers its embedding through totaling node data above the local network area neighborhood of a element node.

A critical contrast among our methodology and conventional Graph Neural Networks is nothing but edge loads in the graph are not provided as feed in. Generally, we fix a utilizing client-specific connection scoring function which is prepared through a directed design. Though, the additional pliability of edge loads creates the learning procedure susceptible to overfitting, as the lone source of administered signal for the relation scoring function comes from client element collaborations (that are generally inadequate). To solve this particular issue, we build up a strategy for uniformity of edge loads in the learning procedure, which promotes improved reasoning. We build up a methodology dependent on LS, which expects that adjoining elements in the $\mathrm{KG}$ are probably going to have comparable client significance label/scores. In the setting, this supposition implies that clients in general have identical inclinations to elements that are close by in the knowledge graph. The KAG neural networks along with LS uniformation can be brought together in a similar structure, where LS can be viewed as a normal decision of uniformation on KAG. Examinations show that our strategy accomplishes critical gains over cutting edge strategies in recommendation exactness. We likewise show that our technique keeps up solid recommendation execution in the cold-start situations where client element communications are scanty.

\section{RELATED WORK}

- Recommendations System

MOVREC is a recommender system for movies presented by D.K. Yadav et al. based on a collaborative filtering approach. Collaborative filtering uses userprovided information. A movie is recommended to users who are structured in a way with the highest rating first, based on the review of the information given. Two conventional recommendation systems have also been studied by Luis M Capos et al, i.e. collaborative filtering and filtering based on content. $\mathrm{He}$ introduced a new method, which is a mixture of collective filtering and the Bayesian network, since both methods have their own disadvantages. Harpreet Kaur et al. have suggested a hybrid framework. This approach utilizes a content mix along with a collaborative algorithm that filters. The history of the films is often taken into account when recommending a movie. A major role in the Recommendation is the interaction between the user - item relationship and the user - user relationship. A cluster is created using chameleon by clubbing item specific details or user specific information via Utkarsh Gupta et al. This is a systematic approach Hierarchical clustering dependent for the proposed technique. To predict the ranking, a voting system for objects is used. This system Hierarchical clustering dependent has a lower level of error and better clustering of associated objects. Clustering has been suggested as a way of coping with Urszula $\mathrm{Ku}$ 'zelewska et al recommended structures. Two approaches were introduced and tested by members of the computing cluster. As a basis for comparing the usefulness of the two suggested approaches, collaborative memory-based filtering and centroid-based solution methods were used. Compared with only a centroid based approach, the combination resulted in a significant increase Even in order of the 


\section{SJIF Impact Factor 2021: 8.013| ISI I.F.Value:1.241| Journal DOI: 10.36713/epra2016 ISSN: 2455-7838(Online) EPRA International Journal of Research and Development (IJRD)}

suggestions produced. CostinGabriel Chiru et al, which uses the user's data to provide movie reviews, suggested a Movie Recommender scheme. The purpose of this scheme is intended to solve the particular problem of resulting from overlooking user-specific data. This collects the psychological profile, the history of the user and the data from other websites about movie ratings. It is a model of a hybrid that uses both collaborative sorting approaches and filtering based on content. Hongli LIn et al, In order for each trainee to predict the difficulty level of each scenario. Suggested a method called content boosted collaborative filtering. The algorithm is split into two phases, In particular, the content-based filtering that enhances the present case ratings information for trainees and the second is a joint filtering that gives the final predictions. Not only does this $\mathrm{CBCF}$ algorithm include the benefits of both $\mathrm{CF}$ and $\mathrm{CBF}$, but it also overcomes both of their disadvantages.

\section{- Recommendations with Knowledge Graphs}

Current Knowledge graph-aware recommender systems are divided in 3 groups:

$\{1\}$ Embedding-built approaches initially process a KGE algorithm before incorporating learned entity embedding into the recommendation. The KGE algorithms emphasis more on modelling rigorous semantic likeliness (example: Trans-E [2] meaning head + relation=tail), making them more appropriate for graph applications like link forecast instead of recommendations. Furthermore, embedding-based approaches often lack as start-to-end training process.

$\{2\}$ Path-built approaches look at different patterns of interactions between entities in a knowledge graph to help recommendations with added direction. Pathbased approaches allow more intuitive use of KGs, but they depend heavily on manual created graphs, that are difficult to adjust in practice.

\{3\} Hybrid approaches incorporate the above two methods and use the structure of KGs to learn user/item embedding. The model we propose can be an example of hybrid methodology.

\section{PROBLEM FORMULATION}

We start by introducing notation and defining the KG-aware recommendations issue. We have a set of users $\mathbf{M}$ and a set of items $\mathbf{N}$ in a standard recommendation scenario. The client-item interaction medium $\mathbf{P}$ is determined by client's implied input, with $\mathrm{pmn}=1$ indicating that user $\mathrm{M}$ has interacted with item $\mathrm{N}$ in a way, like click, view, and buy. We have a knowledge graph $\mathbf{D}=(\mathrm{h}, \mathrm{r}, \mathrm{t})$, where $\mathrm{h} \in \mathrm{E}, \mathrm{r} \in \mathrm{R}$, and $\mathrm{t}$

\begin{tabular}{|l|l|}
\hline Symbol & Meaning \\
\hline $\mathrm{M}=\{\mathrm{u} 1, \ldots\}$ & Bunch of users \\
\hline $\mathrm{N}=\{\mathrm{v} 1, \ldots\}$ & Bunch of items \\
\hline $\mathrm{P}$ & User-item interaction medium \\
\hline $\mathrm{D}=(\mathrm{E}, \mathrm{R})$ & $\mathrm{KG}$ \\
\hline $\mathrm{E}=\{\mathrm{e} 1, \ldots\}$ & Bunch of entities \\
\hline $\mathrm{R}=\{\mathrm{r} 1, \ldots\}$ & Bunch of relations \\
\hline $\mathrm{E} / \mathrm{N}$ & Bunch of non-item entities \\
\hline
\end{tabular}

$\in \mathrm{E}$ mean the head, relation, and tail of a knowledge triplet, respectively, while $\mathrm{E}$ and $\mathrm{R}$ mean the set of entities $\mathrm{E}$ and relations $\mathrm{R}$ in the KG. In instance; the triple (Red dragon, film.film.star, Brett Ratner) indicates that Brett Ratner is the main character in

"Red dragon". In certain suggestion cases, an item $v \in$ $\mathrm{V}$ relates to an entity $\mathrm{e} \in \mathrm{E}$ (for example, in MovieLens, the element " Red dragon" also appears as an entity in the knowledge graph). Item $\mathrm{N}(\mathrm{N} \subseteq \mathrm{E})$ as well as non-items E\N (example: nodes equivalent to item/product qualities) make up the set of entities E. Our goal is to forecast whether user $\mathrm{M}$ would be interested in item $\mathrm{N}$, that he or she had not previously interacted with, based on the user-item interaction medium $\mathrm{P}$ and the knowledge graph $\mathrm{D}$. We want to learn a prediction function $\mathrm{p}^{\wedge} \mathrm{mn}=\mathrm{Z}(\mathrm{m}, \mathrm{n} \mid \Theta, \mathrm{P}, \mathrm{D})$, where $p^{\wedge} \mathrm{mn}$, where $\mathrm{p}^{\wedge} \mathrm{mn}$ mean the likelihood that user $\mathrm{M}$ will interact with item $\mathrm{N}$ and $\Theta$ are model parameters of function $\mathrm{z}$.

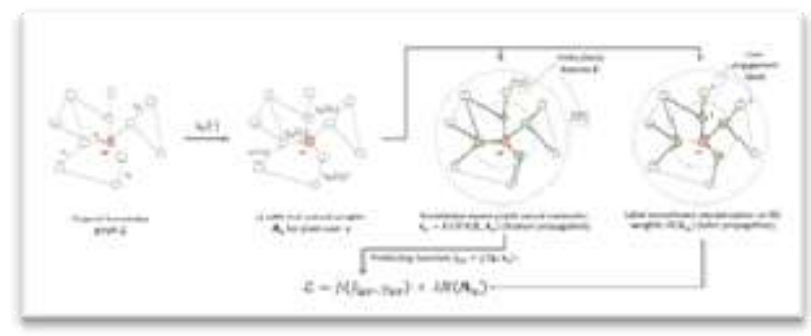

Label Smoothness Regularization

(An ingredient of higher model accuracy)

When we use the cross-entropy loss to solve a classification problem, we expect true labels to have 1 and false labels to have 0 . To put it another way, we are certain that the real labels are true, while the others are not. Is this always the case? May be not. Many manual annotations are the product of collaboration between several participants. They may have different standards. They can make some errors. After all, they are human. 


\section{SJIF Impact Factor 2021: 8.013| ISI I.F.Value:1.241| Journal DOI: 10.36713/epra2016 ISSN: 2455-7838(Online) EPRA International Journal of Research and Development (IJRD)

As a consequence, the ground truth labels on which we have perfect values could be incorrect. We may, for example, reduce the loss target values from 1 to 0.9 . As a result, we marginally boost the goal value of 0 for the others. This is nothing but Label smoothing.

The arguments in cross entropy loss, as defined by Tensorflow, are as follows:

\section{tf.losses.softmax_cross_entropy(} onehot_labels, logits, weights $=1.0$, label_smoothing $=0$, scope $=$ None, loss_collection=tf.GraphKeys.LOSSES, reduction=Reduction.SUM_BY_NONZERO_WE

)

This is how the label smoothing argument is defined in the Tensorflow documentation

If label_smoothing is nonzero, smooth the labels towards 1/num_classes: new_onehot labels = onehot labels * $(1-$ label smoothing $)+$ label smoothing / num classes

What it means?

For example, you were training a model for binary classification. Your labels could be $0-$ cat, $1-$ not cat.

Now, suppose you label_smoothing is 0.2

By using above equation, we get:

new_onehot_labels $=\left[\begin{array}{ll}0 & 1\end{array}\right] *(1-0.2)+0.2 / 2=[0$ $1] *(0.8)+0 . \overline{1}$ new_onehot_labels $=\left[\begin{array}{ll}0.9 & 0.1\end{array}\right]$

Instead of hard labels, which are 0 and 1 , these are soft labels. As a result, when an incorrect prediction occurs, you will suffer a smaller loss, and the model will be penalize and learn incorrectly to a lesser extent.

Label smoothing, in essence, will assist the model in training around mislabeled data, thereby improving its robustness and efficiency.

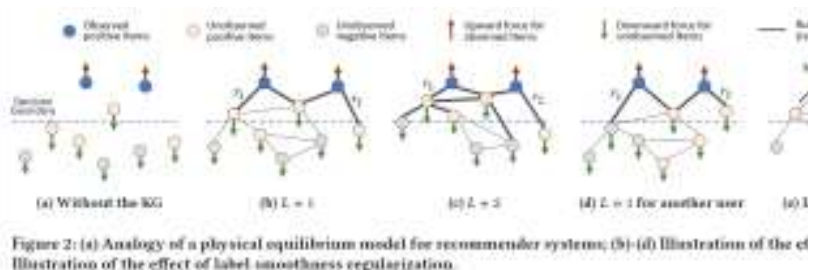

\section{DISCUSSION}

What role does the knowledge graph play in identifying user's interests? To understand this, we can make an analogy with the physical symmetry model as in Fig 2. Each entity/item is viewed as a unit, with the administrated positive client-relevancy signal acting as a power pushing the unobserved items down and the negative items signal acting as a power pulling the noted positive items up from the decision borderline. These items are very lightly connected with one another without $\mathrm{KG}$ (Figure 2a) due to the collaborative filtering effect (which, for the sake of consistency, is not drawn here). Edges in the KG, on the other hand, function as rubber bands, enforcing explicit limits on associated individuals. When the number of layers is $\mathrm{L}$ $=1$ (Figure 2b), each entity's representation is a mix of itself and its adjacent neighbours; thus, focusing on the positive things would bring their adjacent neighbours up together as well. As L increases, the upward force in the $\mathrm{KG}$ increases, which aids in the exploration of user's long-distance needs and the retrieval of more positive elements (Figure 2c). It's also worth noting that the KG's proximity restriction is tailored because the rubber band's strength (i.e., su (r)) is user- and relationspecific: 1 user might choose relation r1 (Figure 2b), whereas another might prefer relation r2 (Figure 2d). Edge loads could be assigned incorrectly, for e.g, too small to pull up unobserved objects, despite force exerted by edges in the knowledge graph (i.e., rubber bands being too weak). The label smoothness assumption is then used to help regularize the learning of edge weights, as shown in Figure (2e). Assume we have the positive model in the left and aim to replicate its label with the remaining items. As the true relevant label of the held-out model is one and the right model has the highest label rate, the Label Smoothness uniformation term $\mathrm{U}(\mathrm{A})$ will push the edges with projectiles to be as wide as possible so that the label would "flow" as much as possible from the blue to the striped sample. As a result, the rubber bands (depicted by projectiles) will tighten and the model will be more inclined to pull up the two upper pink objects.

\section{Datasets}

The MovieLens-20M dataset, which comprises of roughly twenty million clear ratings (From 1-5) on the MovieLens website, is a commonly used benchmark dataset in movie recommendations. There are 102,569 elements, 499474 edges and 32 relationcategories in the KG. 


\section{SJIF Impact Factor 2021: 8.013| ISI I.F.Value:1.241| Journal DOI: 10.36713/epra2016 ISSN: 2455-7838(Online) EPRA International Journal of Research and Development (IJRD)

\section{RESULTS \\ Efficacy of LS Regularization}

Does the suggested c regularization effective in enhancing GNN status? The dimension of unseen layers to see how output changes to test the efficacy of LS regularization.In recommender systems, Label smoothness regularization can help with learning the edge loads in a knowledge graph and achieving improved simplification.

\section{Results in cold-start scenarios}

One of the main goals of using knowledge graphs in recommender frameworks is to address the problem of scantiness. We varied the proportions of the training set of MovieLens-20M from $\mathrm{r}=100$ percent to $r=20 \%$ to investigate the efficiency of KG-LS in coldstart scenarios (while the validation and test set are kept fixed). As $\mathrm{r}=20 \%$, AUC drops by $8.4 \%, 5.9 \%, 5.4$ percent, $3 . \%, 2.8 \%$, and $4.1 \%$ for the six baselines, respectively, relative to the model trained on complete training data $(r=100 \%)$, but KG-LS only drops by $1.8 \%$. This shows that even when user-item interactions are scarce, KG-LS retains predictive efficiency.

\section{CONCLUSION AND FUTURE WORK}

In this paper, we propose a recommendation system based on a knowledge-aware graph with mark smoothness regularization. KG-LS applies GNN engineering to knowledge graphs by combining neighborhood data with varied loads and using clientspecific relation scoring functions. Furthermore, for learning the edge loads in KGs, the proposed LS restriction and leave-one-out loss provide good uniformity. We also go into how KGs can help recommender systems learn the edge weights and how label smoothness can help with that. The use of LS regularization for recommendation tasks with KGs is suggested in this paper. Additional graph tasks, like relation prediction and node sorting, are important to investigate using the LS assumption. A fruitful way is to look into the theoretical relationship among feature propagation and label propagation.

\section{REFERENCES}

1. Ram D. Gopal Rajkumar Venkatesan Fang Yin Bhavik Pathak, Robert Garnkel.Empirical analysis of the impact of recommender systems on sales, journal of man-agement information systems december 2014.

2. Hannes Werthner L'aszl'o Grad-Gyenge, Peter Filzmoser. Recommendations on a knowledge graph 2015.
3. Kevin Shah Jeet Gandhi-Vrushal Kamtikar November 2016 Rupali Hande, Ajinkya Gutti. Moviemender a movie recommender system. International Journal of Engineering Sciences and Research Technology (IJESRT), ISSN 22779655, November 2016.

4. William Cohen Rose Catherine. Personalized recommendations using knowledge graphs: A probabilistic logic programming approach 2017.

5. Thomas N Kipf and Max Welling 2017. Semisupervised classification with graph convolutional networks. In the 5th international conference on learning representations. 